\title{
Adaptability Analysis of the Software FLUX Calculating Ship Magnetic Field
}

\author{
J.H. Cao \\ Naval Academy of Armament \\ Beijing, 100161, China
}

\author{
H.F. $\mathrm{Mu}$ \\ Naval Academy of Armament \\ Beijing, 100161, China
}

\author{
B. Zhuang \\ Naval Academy of Armament \\ Beijing, 100161, China
}

\begin{abstract}
To deal with the large calculation error of ship magnetic field, this paper simulated the submarine magnetic field based on the software FLUX. Submarine's geometry modelling, meshing and magnetic field calculation have done in order. According to magnetic field data and images, comparative analysis of calculation results by different finite element methods have done. The attentions from geometric modelling, meshing and finite element calculation were separately listed. Simulation results show that FLUX can more accurately calculate the submarine magnetic field. Experimental results provided that the software FLUX was an excellent software for calculating ship magnetic field.
\end{abstract}

Keywords- ship magnetic field; finite element calculation; software FLUX

\section{INTRODUCTION}

The accurate prediction of ship magnetic field is of great significance for the ship's magnetic hiding performance. At present, several methods of ship magnetic field prediction are often used, which are respectively mother ship's conversion, empirical formula calculation, magnetic ship model test and numerical simulation[1-2]. Among them, the method of mother ship's conversion requires harsh conditions; the actual application generally needs correction in combination with other methods, such as empirical formula, physics simulation, simulation, etc. The method of empirical formula calculation is mainly dependent on the experience of staff. As the staffs use the method to calculate, there may be more human disturbance factors. The method of magnetic ship model test is currently widely used, which introduces high demands for making magnetic model, in addition to its long construction period, poor universal property, high cost of testing. The method of numerical simulation has rapid, simple and economical advantages. With the deepening of the study, the applications of the numerical simulation method in the ship magnetic field computing are increasingly widespread. Especially the mature calculation of various commercial softwares provides a basic simulation platform for the calculation of the ship magnetic field. This paper has calculated the submarine's magnetic field by the software FLUX.

\section{Finite ELEMENT THEORY FOR SHIP MAgNetic FIELD}

Finite element method is based on the variational principle or weighted residuals method, combined with element meshing and piecewise interpolation, a discrete numerical method is used to solve mathematical equations. Finite element method represents the entire contiguous area with many subdomains, where unknown function is expressed by simple interpolation functions with unknown coefficients.

2D magnetostatic problems use magnetic vector potential and 3D magnetostatic problems use magnetic scalar potential. The scalar magnetic potential has some advantages than the magnetic vector potential in computing memory, required memory and uniqueness of results, which is critical for ship magnetic field calculation [1]. The general equation of magnetic scalar model for finite element method is as follows:

$$
\operatorname{div}\left(\mu_{r} \mu_{0}(-\operatorname{grad} \phi+\vec{T})+\vec{B}_{r}\right)=0
$$

In formulation (1), $\phi$ is the scalar magnetic potential, $-\operatorname{grad} \phi$ is the irrotational component of the magnetic field $\vec{H}$ (that is $\operatorname{rot}(\operatorname{grad} \phi)=0) . \vec{T}$ is zero or rotational component.

When the current source does not need to mesh, partial scalar magnetic potential $\phi_{r e d H_{j}}$ is used. $\phi_{r e d H_{j}}$ is relevant with $\vec{H}$, which is the analytical solution of Biot-Savart formula. $\vec{T}=\vec{H}_{j}$ in the scalar model of general solution.

$$
\vec{H}=-\operatorname{grad} \phi_{\text {redH }_{j}}+\vec{H}_{j}
$$
form:

Then the solving equation can be written as the following

$$
\operatorname{div}\left(\mu_{r} \mu_{0}\left(-\operatorname{grad}\left(\phi_{r^{2} H_{j}}+\vec{H}_{j}\right)\right)+\vec{B}_{r}\right)=0
$$

The state variable is part of the magnetic scalar potential $\phi_{r_{e d H}}$ which is associated with $\vec{H}$. When the coil current or 
uniform external magnetic field exists, scalar magnetic potential $\phi_{\text {redH }_{j}}$ can used.

The literatures [3-4] used the finite element method respectively to analyze the ship's magnetic field and the submarine's magnetic field. The literatures [5-6] on studied the thin ferromagnetic objects. These used line unit and face unit to treat 2D and 3D problems, which can greatly reduce the number of element and the computing time while compared with the traditional finite element methods. The above research results have been applied to ship magnetic field computation. The literatures [7-8] used the geometric transformation method based on finite element method, which made the open domain problem transformate into a closed domain problems. So this method greatly facilitate the calculation of magnetic field. Now the method has been applied to ship magnetic field calculation. The literature [9] studied the applicability of simulation of ship degaussing coil by using finite element method.

\section{INTRODUCTION OF FLUX SOFTWARE}

FLUX is now widely used in ship magnetic field calculation by USA and France. FLUX software can better handle as follows: ships complex shape, inconsistencies between hull thickness and hull large-scale, open field magnetic field calculation. FLUX software is based on the following assumptions: the normal component of the magnetic field in thin-shell region is zero or negligible, so the scalar magnetic potential in thickness can be considered to be constant. FLUX uses surface domain of material properties and thickness parameters to substitute for body domain with geometric thickness, as shown in Figure 1, which improves the calculation efficiency.

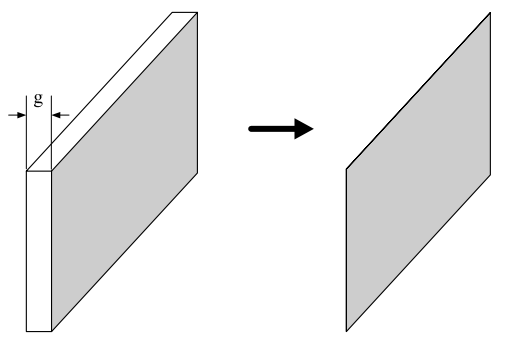

FIGURE I. DIAGRAM OF THIN SHELL SIMULATION

Calculating magnetic field of FLUX software consists of the following steps:

Step 1: to select model type according to calculation problem;

Step 2: geometric model can be directly built in FLUX software. Geometric model can also be imported though the third-party software, then need the geometry check and repair;

Step 3: to mesh on geometric model;

Step 4: to set physical properties;

Step 5: to calculate based on previous settings;

Step 6: the post processing of calculation results.

\section{Calculation Example of Submarine Magnetic FIELD}

Base on FLUX platform, submarine's magnetic field by finite element simulation is calculated and compared.

\section{A. Geometry Modelling}

Because the ship magnetic field is calculated and analysis at a certain distance, according to simplification principles for magnetic field[10-11], so the complex shapes of the submarine is simplified and geometric model is built based on the FLUX platform, as shown in Figure 2. The following errors should be avoided in geometric modeling process that the crossing between line and line, the crossing between line and face, the crossing between face and face, and so on. Figure 3 shows that geometric modeling error of submarine rudder is caused by line-face intersection, which lead to some volumes not to generate. As shown in Figure 4 is the rudder of the internal face ( the error face in Figure 4) not set to No Exist, resulting in errors during volume meshing. FLUX software requests infinite box's size should be at least twice times larger than the model's size [12], as shown in Figure 5.

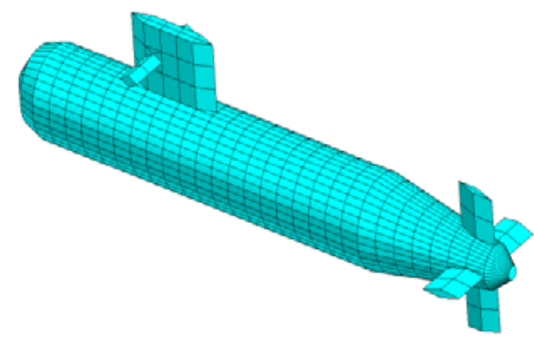

FIGURE II. SUBMARINE'SGEOMETRY MODEL

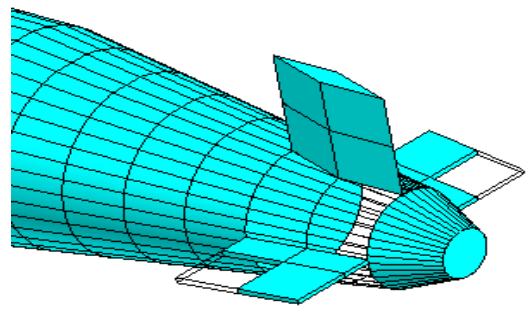

FIGURE III. CROSSING BETWEEN LINE AND FACE LEAD TO SOME VOLUMES NOT BUILD.

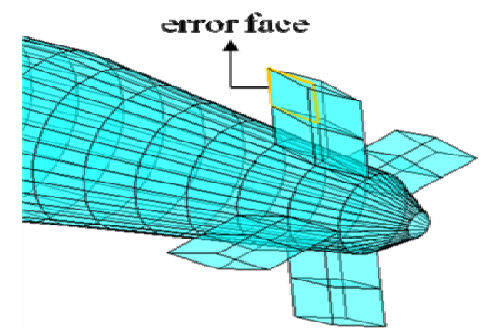

FIGURE IV. NO STRUCTURAL FACE ISN'T SET TO NO EXIST. 


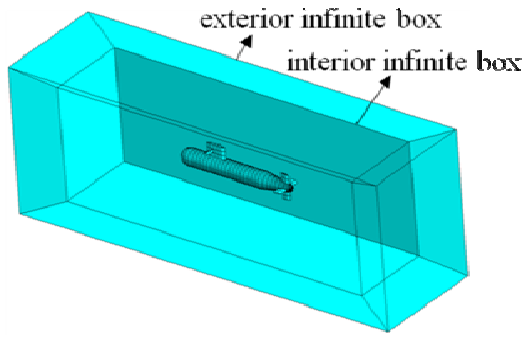

FIGURE V. INFINITE BOX IN FLUX SOFTWARE.

\section{B. Meshing}

FLUX is through the size and quantity of meshing elements to control and adjust the meshing. Element sizes are controlled through setting points and element numbers are controlled through setting lines. Ship shape is very complex. Automesh of only tetrahedral element is relatively simple. So the submarine in the paper is meshed by automesh. Meshing result is shown in Figure 6.

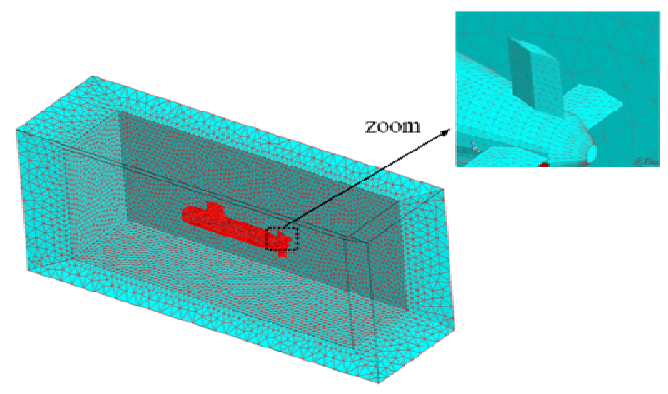

FIGURE VI. MESHING OF SUBMARINE AND INFINITE BOX.

\section{Physical Properties}

Ship magnetic field belongs to magneto static problem, so the model of magneto static 3D and magnetic scalar potential formulations was used to calculate a submarine magnetic field. Because earth's magnetic field is weak magnetic field, so magnetization curve is defined as a linear curve, and relative permeability is defined as a unique magnetic properties of materials.

\section{Magnetic Field Calculation}

FLUX software can be parameterized calculated. FLUX software can be used first-order and second-order finite element method. In the same meshing conditions, calculation through second-order finite element method can provide more accurate results, and less time. Figure $7 \sim 8$ were calculated magnetic field of submarine respectively though first-order and second-order finite element method.

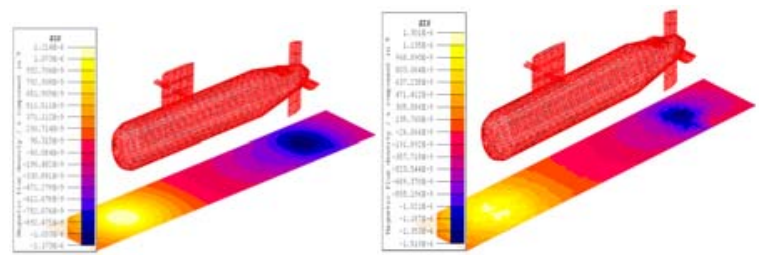

FIGURE VII. MAGNETIC FIELD COMPONENT. ZIX (LEFT: 1ST ORDER ELEMENT, RIGHT: 2ND ORDER ELEMENT).

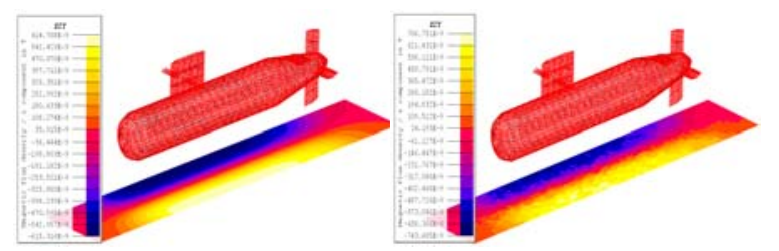

FIGURE VIII

MAGNETIC FIELD COMPONENT ZIZ (LEFT: 1ST ORDER ELEMENT, RIGHT: 2ND ORDER ELEMENT).

By using finite element method for first-order and second-order, the magnetic field at a certain depth under submarine keel and side were calculated sepately, as shown in Figure 9 11. Measuring points of 50 points was uniformly distributed.

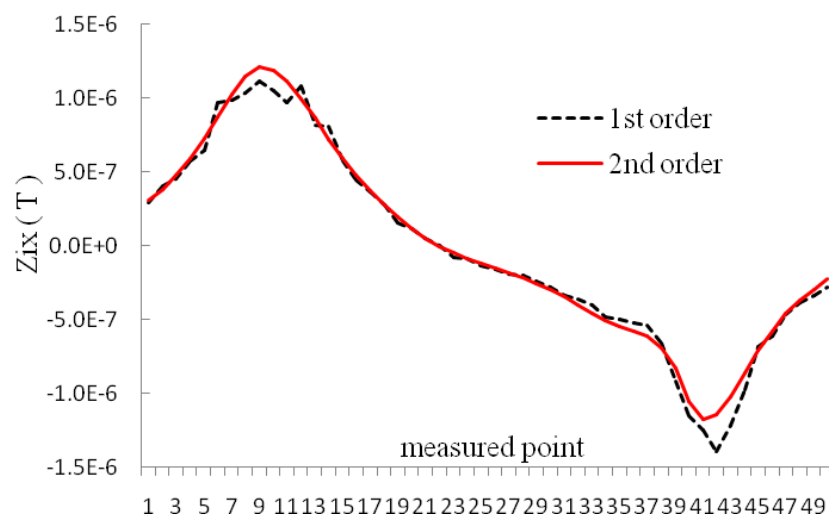

FIGURE IX. CONSTRAST BETWEEN TWO KINDS OF MAGNETIC ZIX UNDER SUBMARINE'S KEEL.

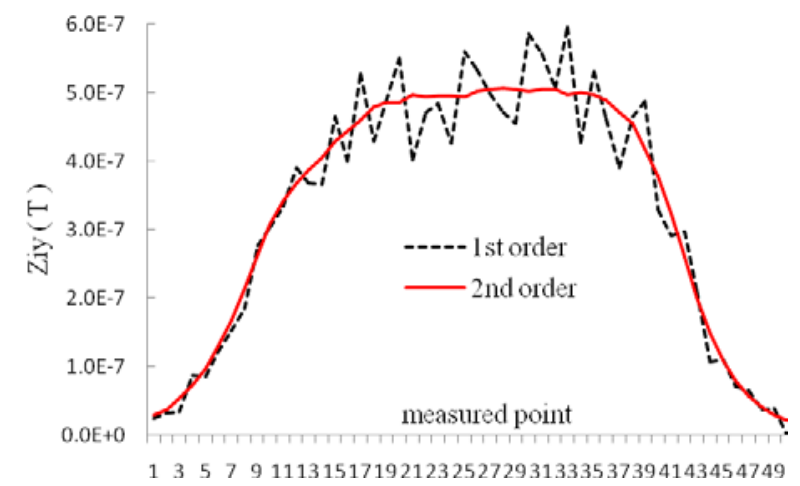

FIGURE X. CONSTRAST BETWEEN TWO KINDS OF MAGNETIC ZIY UNDER SUBMARINE'S SIDE. 


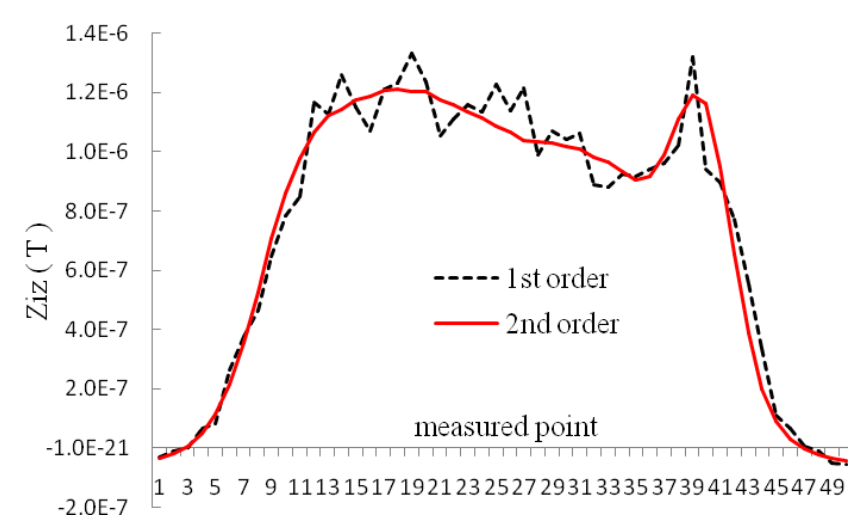

FIGURE XI. CONSTRAST BETWEEN TWO KINDS OF MAGNETIC ZIZ UNDER SUBMARINE'S KEEL.

By comparing analysis in Figure $7 \sim$ Figure 12, the following situation can be seen: when submarine magnetic field was calculated through FLUX software, the three components ( Zix, Ziy and Ziz ) of the magnetic field of submarine has great difference separately by $1^{\text {st }}$ order and $2^{\text {nd }}$ order finite element method. The calculation of the component Ziy and Ziz have large fluctuations by $1^{\text {st }}$ order finite element method. For symmetrical structure like submarine, apparently, the big wave phenomena is the performance of calculation errors. However, the accuracy of magnetic field calculation is better by $2^{\text {nd }}$ order finite element method.

\section{CONCLUSION}

Based on the finite element calculation platform of the electromagnetic software FLUX, the magnetic field of a submarine is studied based on the finite element calculation. In this paper, the following aspects are studied: geometric modelling, meshing, first-order elements and second-order elements of finite element calculation. Experimental results showed that through the correct geometric modelling, and according to the accuracy requirements of the object, using proper meshing method and the second order finite element method, FLUX can accurately simulate the submarine's magnetic field. FLUX software has good adaptability on the magnetic field calculation of submarine. FLUX software can be used in engineering practice.

\section{REFERENCES}

[1] Jiang Zhipeng, Zhuang Biao, Guan Tao, et al. Calculation of Ship Magnetic Fields Based on the Software FLUX[J]. Marine Electric \& Electronic Engineering, 2010, 30(12): 17-20.

[2] Wang De-qiang, Yu Qiang. Overview on the numerical calculation method of ship's magnetic field[J]. Ship Science and Technology, 2014, 36(3): 1-6.

[3] Zhang Xiaohua, Li Youding. Finite element prediction of ship's magnetic field[J]. Mine Warfare \& Ship Self-defence, 1995, 3: 39-43.

[4] Weng Xingtai, Cao Meifen. A Resolution on the Calculation of the Inducted Magnetic field of a Submarine[J]. JOURNAL OF SHANGHAI JIAOTONG UNIVERSITY, 1994, 28(5): 69-76.

[5] Rodger D, Leonard P J, Lai H C. Surface elements for modelling 3D fields around thin iron sheets[J]. IEEE Trans. Mag, 1993, 29(2): 14831486.
[6] Brunotte X, Meunier G. Line element for efficient computation of the magnetic field created by thin iron plates[J]. IEEE Trans. Mag, 1990, 26(5): 2196-2198.

[7] Imhoff J F, Meunier G, Brunotte X, Sabonnadihre J C. An original solution for unbounded electromagnetic 2D and 3D problems throughout the finite element method[J]. IEEE Trans. Mag, 1990, 26(5): 1659-1661.

[8] Brunotte X, Meunier G, Imhoff J F. Finite Element Modeling of Unbounded Problems using Transformations :A Rigorous, Powerful and Easy Solution [J]. IEEE Trans. Mag, 1992, 28(2): 1663-1666.

[9] Le Dorze F, Coulomb J L, Brunotte X. Modeling of Degaussing Coils Effects in Ships by the Method of Reduced Scalar Potential Jump [J]. IEEE Trans. Mag, 1998, 34(5): 2477-2480.

[10] Brunotte X, Meunier G, Bongiraud J P. Ship Magnetizations Modelling by the Finite Element Method [J]. IEEE Trans. Mag, 1993, 29(2): 1970-1975.

[11] Cao Junhong, Zhuang Biao, MU Haifang, et al. An Efficient Modeling and Meshing Method for the Finite Element Calculation of the Ship Magnetic Field[J]. Applied Mechanics and Materials. 2014, (494-495): 767-770.

[12] CEDRAT Company. General Tools Geometry and Mesh [M]. FLUX 9.30 User's guide. 2005 